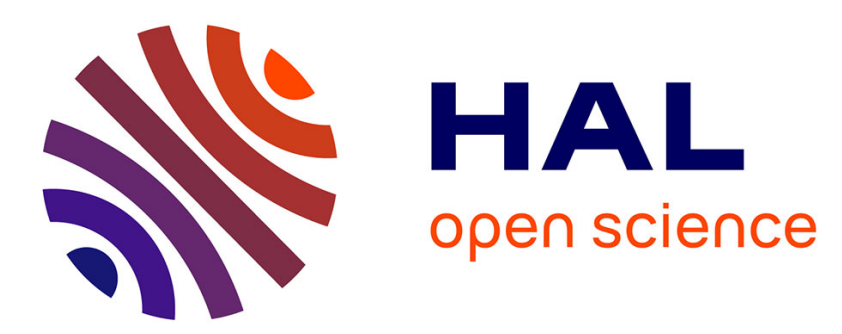

\title{
Etude de la cavitation par caractérisation dynamique impulsionnelle
}

Christophe Delebarre, I. Baquet, Edouard Radziszewski, C. Bruneel, J. Frohly

\section{To cite this version:}

Christophe Delebarre, I. Baquet, Edouard Radziszewski, C. Bruneel, J. Frohly. Etude de la cavitation par caractérisation dynamique impulsionnelle. Journal de Physique IV Proceedings, 1994, 04 (C5), pp.C5-1173-C5-1176. 10.1051/jp4:19945258 . jpa-00252947

\section{HAL Id: jpa-00252947 https://hal.science/jpa-00252947}

Submitted on 1 Jan 1994

HAL is a multi-disciplinary open access archive for the deposit and dissemination of scientific research documents, whether they are published or not. The documents may come from teaching and research institutions in France or abroad, or from public or private research centers.
L'archive ouverte pluridisciplinaire HAL, est destinée au dépôt et à la diffusion de documents scientifiques de niveau recherche, publiés ou non, émanant des établissements d'enseignement et de recherche français ou étrangers, des laboratoires publics ou privés. 


\title{
Etude de la cavitation par caractérisation dynamique impulsionnelle
}

\author{
C. DELEBARRE, I. BAQUET, E. RADZISZEWSKI, C. BRUNEEL et J. FROHLY \\ IEMN, Département OAE, UMR 9929 du CNRS, Université de Valenciennes, Le Mont Houy, BP. 311, \\ 59304 Valenciennes cedex, France
}

\begin{abstract}
Résumé: La cavitation ultrasonore haute fréquence est aujourd'hui de plus en plus étudiée car elle fournit un outil efficace à la synthèse chimique. Le transducteur ultrasonore étant un émetteur récepteur, il est utilisé en mode réflexion. Notre but est d'obtenir des mesures quantitatives de paramètres physiques des bulles en étudiant la propagation d'impulsions ultrasonores dans le milieu de cavitation. L'intérêt majeur de cette méthode réside dans son caractère non perturbant.
\end{abstract}

\begin{abstract}
As it provides a more efficient tool for some typical chemical reactions, the high frequency ultrasonic cavitation is nowadays increasingly studied. The ultrasonic transducer is a reciprocal sensor, so it is used in this work in a pulse reflection mode. Our purpose is to obtain quantitative measurements of physical parameters of the bubbles, studying the propagation of ultrasonic pulses in the cavitating medium.
\end{abstract}

\section{INTRODUCTION.}

Qu'il soit volontairement provoqué ou non, l'état cavitationnel d'un liquide engerdre des phénomènes physiques tels que l'érosion, le nettoyage de surface, la modification de réactions chimiques etc... C'est pourquoi il demeure très étudié dans la littérature. De l'étude de la cavitation causée par les pales d'hélice des navires, à celle induite par les faisceaux ultrasonores focalisés dans les applications biologiques et médicales, nombreux sont les domaines où ce phénomène intervient.

Depuis que les chimistes l'utilisent comme outil de synthèse et de catalyse, la cavitation ultrasonore connait un regain d'intérêt. Toutefois, afin que les résultats expérimentaux des travaux effectués dans ce domaine à des puissances acoustiques et des fréquences différentes puissent être comparés, il est nécessaire de disposer de techniques permettant de caractériser l'état de cavitation. Ainsi, les paramètres associés aux champs de bulles de cavitation (taille et concentration) pourront alors être corrélés aux configurations expérimentales qui servent à les générer.

Le procédé que nous avons mis au point est basé sur l'idée que les bulles de cavitation modifient la propagation d'impulsions ultrasonores dans le milieu devenu diphasique. Il est donc possible de relier cette modification à l'état cavitationnel du liquide.

L'aspect original de ce travail réside dans le fait que le même transducteur est utilisé à la fois pour produire la cavitation et pour la détecter. Ainsi, les résultats ne sont pas biaisés par l'utilisation de plusieurs systèmes ayant des réponses impulsionnelles différentes et le champ acoustique n'est pas perturbé par la présence des dispositifs de mesure. 


\section{CARACTERISATION DYNAMIQUE IMPULSIONNELLE.}

\subsection{Dispositif experimental.}

Le dispositif experimental utilisé dans ce travail est décrit ci-dessous à la figure 1 .

Il s'agit essentiellement d'utiliser un générateur alternatif d'ondes monochromatiques piloté par un générateur d'impulsions. La mesure du taux de distorsion en sortie de l'amplificateur de puissance donne une valeur de $0.5 \%$, soit un écart de l'ordre de $35 \mathrm{~dB}$ entre le fondamental et la première harmonique. Ceci nous permet de nous assurer que toute la puissance électrique se situe dans le fondamental.

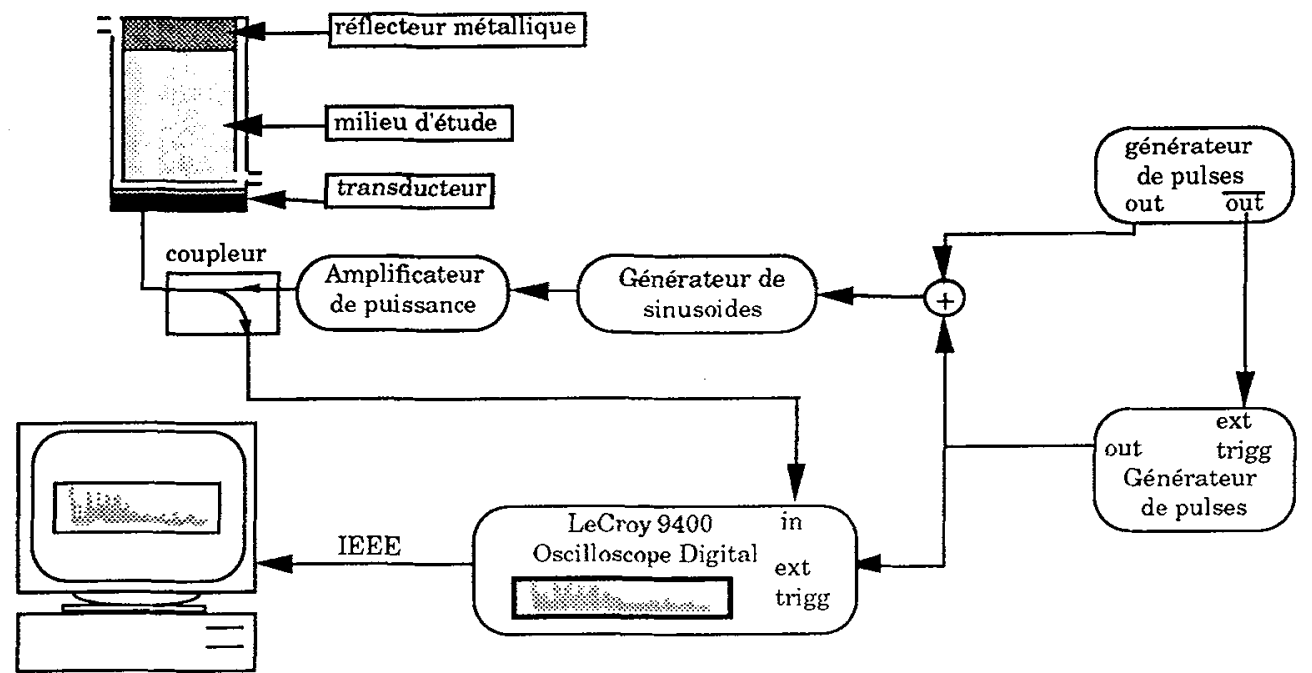

Figure 1: Dispositif expérimental.

Afin de caractériser l'état cavitationnel, nous avons émis l'hypothèse que la présence des bulles dans le fluide devait perturber la propagation d'impulsions ultrasonores. En effet, avec un dispositif semblable à celui qu'utilisent les chimistes, dans lequel les bulles de cavitation peuvent crôtre dans un champ d'ondes stationnaires, la taille des bulles obtenues est de l'ordre de la longueur d'onde.

Il est alors possible d'étudier la propagation d'une impulsion ultrasonore dans le liquide en état de cavitation afin d'obtenir les paramètres quantitatifs permettant une description détaillée du phénomène.

Pour ce faire, le transducteur de puissance est alimenté par un signal composite constitué par la somme d'une impulsion, suffisamment longue pour s'assurer que le régine de cavitation est bien établi, et de la succession d'impulsions d'interrogation très brèves dont on étudie la propagation.

Un réflecteur métallique situé au dessus du réacteur circulaire d'une hauteur de $10 \mathrm{~cm}$ permet d'immobiliser la surface du liquide et donc d'obtenir une réflexion optimale des ondes ultrasonores vers le transducteur.

Dès la fin de l'émission de puissance, les bulles de cavitation en suspension dans le fluide remontent vers la surface. C'est le temps associé à la dissipation des bulles que l'on peut qualifier de durée de relaxation du champ de bulles de cavitation.

Afin d'effectuer une mesure expérimentale de la durée de relaxation, nous avons détecté l'amplitude de 200 impulsions interrogation equiretardées après leur parcours dans le liquide. La représentation de l'amplitude de ces impulsions, en fonction du retard de celles-ci par rapport à l'instant de coupure des ultrasons de puissance, permet d'effectuer la mesure de la constante de relaxation des bulles (figure 2 ). 


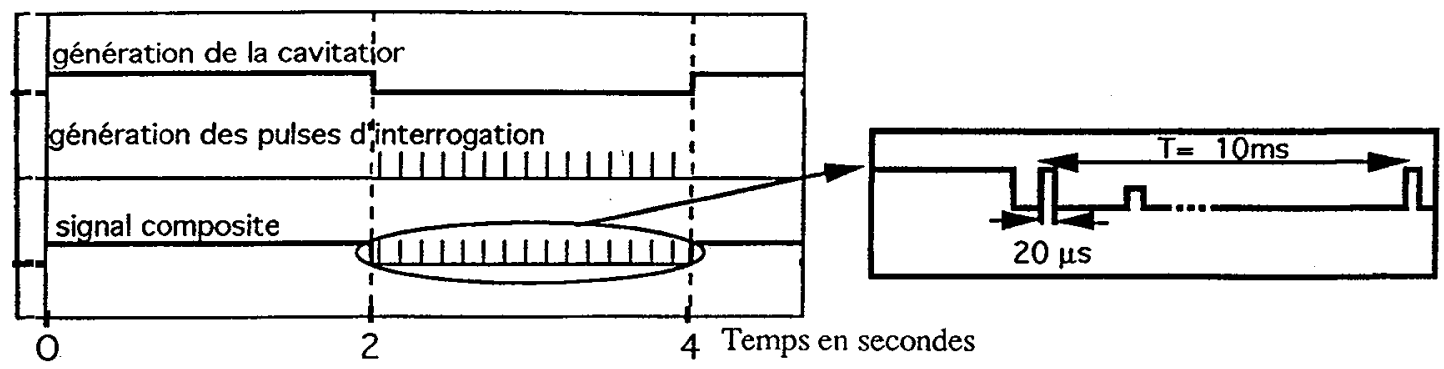

Figure 2: Chronogramme du signal émis.

On peut alors considérer que toutes les bulles sont dissipées lorsque l'amplitude reçue est identique à celle obtenue lorsque la propagation se fait dans le liquide exempt de bulles. Ce procédé d'étude de la cavitation peut être résumé sous le terme générique: caractérisation dynamique impulsionnelle (C.D.I.). Dans un premier temps, nous avons étudié la relaxation d'un champ de bulles dans l'eau [1]. Puis, afin de valider notre méthode de mesure, nous avons fait varier les caractéristiques du milieu diphasique (fluide et bulles de cavitation). Deux études ont alors été menées: l'une visant à détecter une variation de répartition en taille des bulles et l'autre à évaluer l'influence de la viscosité du liquide sur la mesure.

\subsection{Application de la CDI à l'étude de la cavitation dans l'eau.}

Ces travaux visant à être utilisés dans le cadre de la sonochimie, le milieu d'étude que nous avons choisi comme référence est l'eau distillée. La fréquence de travail choisie est de $450 \mathrm{KHz}$.Les impulsions d'interrrogation durent 20 microsecondes et sont distantes de 10 millisecondes. La courbe représentant l'enregistrement de l'amplitude reçue pour chacune des implusions successives est tracée en figure 3.

Le temps de dissipation des bulles dans l'eau est de 600 millisecondes.

D'après les travaux de COMOLET [2] , la vitesse limite d'ascension d'une bulle rigide est donnée par:

$$
\mathrm{U}=\frac{2}{9} \frac{\rho \mathrm{g} \mathrm{I}_{0}^{2}}{\mu}
$$

avec $\rho$ qui représente la masse volumique du liquide, $g$ l'accélération de la pesanteur, $\mathrm{r}_{0}$ le rayon de la bulle et $\mu$ la viscosité dynamique du liquide

Dans le cas qui nous intéresse, le diamètre des bulles n'excèdent pas 3 millimètres cette loi peut donc parfaitement être utilisée. La vitesse d'ascension dépendant du rayon de la bulle, l'étude du temps d'ascension de toutes les bulles permet donc d'obtenir une donnée caractéristique directement liée à la configuration du champ de bulles (taille des bulles, concentration en bulles, position dans le réacteur).

Pour vérifier cette hypothèse, nous avons appliqué la caractérisation dynamique impulsionnelle à l'acétone qui donne naissance à un nuage de bulles dont les caractéristiques sont différentes de celui obtenu avec de l'eau. L'influence de la viscosité du liquide sur le temps de relaxation des bulles a été mise en évidence en utilisant des solutions de Dextran de concentrations différentes.

3. Etude par CDY de l'influence des paramètres physico-chimiques du liquide sur le temps de dissipation des bulles.

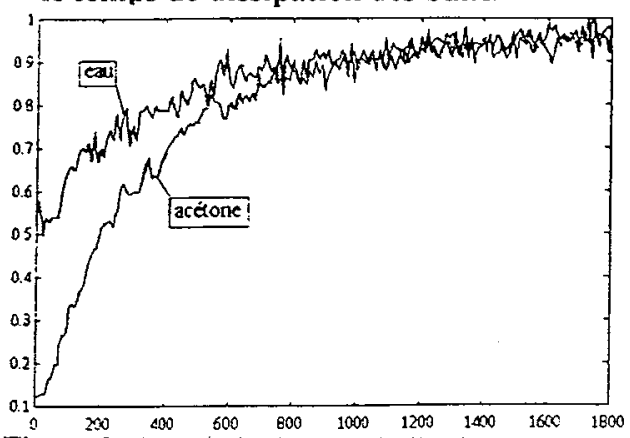

Figure 3 : Etude de l'eau et de l'acétone. absc. $=$ temps en ms Ordon.=amplitude relative reçue

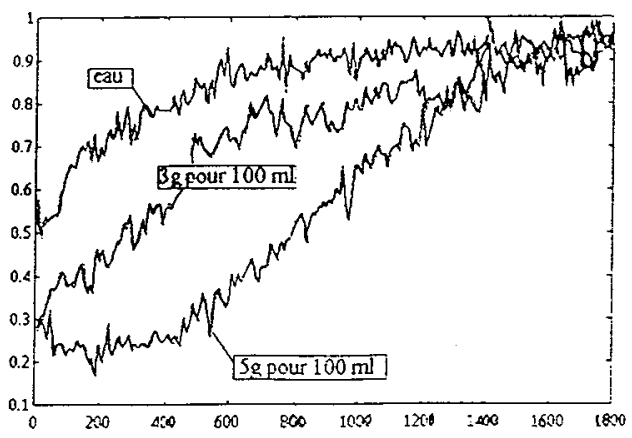

Figure 4: Etude de l'influence de la viscosité absc: $=$ temps en ms Ordon.=amplitude relative reçue 


\subsection{Influence des paramètres régissant la taille des bulles.}

Les bulles de cavitation croissent par diffusion rectifiée pendant les différentes phases du cycle de l'onde de pression [3]. Pendant les phases de compression, la pression du contenu de la bulle ( gaz en suspension + vapeur) est est élevée et le gaz diffuse de la bulle vers le liquide.Pendant les phases de dilatation, la pression dans la bulle est faible et le gaz en suspension dans le liquide diffuse vers l'intérieur de la bulle. Toutefois, la surface d'échange est plus grande pendant les phases de dépression que pendant les phases de compression. Une bulle soumise à une onde acoustique grossit donc, par transfert de masse, à chaque cycle acoustique.

Sa tension superficielle étant plus basse que celle de l'eau, le transfert de masse à travers la paroi d'une bulle est favorisé lorsque celle-ci est immergée dans l'acétone. De plus, la température de vaporisation de l'acétone est plus faible que celle de l'eau. Ces deux facteurs contribuent à générer des bulles plus nombreuses et plus grosses dans l'acétone que dans l'eau.

Par ailleurs, la viscosité dynamique de l'acétone est proche de celle de l'eau. Donc, le temps de dissipation des bulles dans l'acétone ne diffère de celui de l'eau que parce que le champ de bulles possède des caractéristiques différentes (figure 3).

La constante de temps de relaxation des bulles ne change pas de manière significative entre l'eau et l'acétone. Seule l'amplitude est fortement affectée par la concentration importante de bulles.

\subsection{Influence de la viscosité du liquide sur le temps de relaxation des bulles.}

Les variations de la viscosité on été obtenues en utilisant deux solution de Dextran (un sucre de masse molaire élevée) à $3 \mathrm{~g}$ pour $100 \mathrm{ml}$ d'eau et à $5 \mathrm{~g}$ pour $100 \mathrm{ml}$ d'eau dont les viscosités dynamiques mesurées sont respectivement de 4,5 $\mathrm{mPa}$.s et $10 \mathrm{mPa}$.s. La mesure de viscosité a permis de déterminer que les deux solutions peuvent être considéres comme des fluides newtoniens. Le choix de ces concentration est basé sur le fait que la viscosité du liquide doit rester suffisamment faible pour ne pas inhiber la mise en place de la cavitation. Les résultats sont présentés en figure 4.

La viscosité du milieu modifie fortement les constantes de relaxation des bulles pour les différents échantillons.

\section{Conclusion générale.}

L'utilisation de la caractérisation dynamique impulsionnelle montre clairement la possibilié de caractériser l'état de cavitation. En effet, elle permet d'obtenir des mesures quantitatives des paramètres physiques des bulles de cavitation tels que la constante de relaxation des bulles, qui est corrélée à leur taille et leur concentration.

De plus, les résultats présentés dans cet article montrent que la caractérisation dynamique impulsionnelle est un outil assez sensible pour détecter les variations de configuration du champ de bulles liée aux paramètres physico-chimiques du milieu.

Cependant, une approche théorique de l'étude de la propagation d'une impulsion en régime non linéaire reste à faire. Cette approche est en cours de traitement dans notre équipe. Les résultats expérimentaux présentés ici doivent permettre de développer un modèle théorique pertinent.

\section{Notes et Références.}

[1] Delebarre C., "Dynamic characterization of high frequency ultrasonic cavitation" , Ultrasonic symposium 1993 à Vienne (manuscrit en cours de publication)

[2] Comolet R. , Journal de mécanique appliquée, 3, n²2, pp 145-171, 1979

[3] Crum L.A., Ultrasonics, 22 , 215 , 1984. 